\title{
Otomotiv Endüstrisinde Topoloji Optimizasyonu ile Ağırlık Azaltma Uygulaması Üzerine Bir Araştırma
}

\author{
Funda Kahraman $^{1 *}$, Mehmet Küçük ${ }^{2}$ \\ 1* Tarsus Üniversitesi, Mühendislik Fakültesi, Makina Mühendisliği. Bölümü, Tarsus, Türkiye (ORCID: 0000-0002-1661-3376), fkahraman@ tarsus.edu.tr \\ 2 Tarsus Üniversitesi, Lisansüstü Eğitim Enstitüsü, İmalat Mühendisliği ABD, Tarsus, Türkiye (ORCID: 0000-0001-9196-0784), mehmetkucuk1985@gmail.com
}

(İlk Geliş Tarihi 2 Eylül 2020 ve Kabul Tarihi 8 Kasım 2020)

(DOI: $10.31590 /$ ejosat.789424)

\begin{abstract}
ATIF/REFERENCE: Kahraman, F. \& Küçük, M. (2020). Otomotiv Endüstrisinde Topoloji Optimizasyonu ile Ağırlık Azaltma Uygulaması Üzerine Bir Araştırma. Avrupa Bilim ve Teknoloji Dergisi, (20), 623-631.

\section{Öz}

Son yıllarda; otomotiv sektöründeki firmalar, taşıtlarda yakıt tüketimini azaltacak ve enerji verimliliğini arttıracak yeni çalışmalara yönelmişlerdir. Araçların yakıt tüketimini ve buna bağlı olarakta emisyon oranlarını azaltmada en etkili çözümlerden biri taşıt ağırlığında azaltılma yapılmasıdır. Tasarımı yapılan taşıt parçalarında malzeme dağılımının en iyi şekilde sağlanması için optimizasyon yöntemleri kullanılmaktadır. Yapısal optimizasyon ile tasarlanan taşıt parçalarının; boyut, şekil ve topoloji olarak en iyi hale getirilmesi amaçlanır. Yapısal optimizasyonun hedefleri; ağırlı̆̆ı azaltmak, rijitliği arttırmak, stresi azaltmak, üretilebilirliği sağlamak, üretim maliyetlerini düşürmek olarak sıralanabilir. Topoloji optimizasyonu, uygulamalı matematik alanında geliştirilen bir yapısal optimizasyon yöntemidir. Topoloji optimizasyonu; makine, imalat, otomotiv, inşaat ve havacılık gibi birçok disiplinde uygulama alanı bulmaktadır. Bu makale, otomotiv endüstrisinde topoloji optimizasyonu ile ağırlık azaltma çalışmalarına genel bir bakış sunmaktadır. Standart optimizasyon yaklaşımlarına alternatif olarak topoloji optimizasyon yöntemi, verilen performans hedefleri açısından araç ağırlığını azaltmayı ve en uygun malzeme dağılımını bulmayı amaçlayan bir yaklaşımdır. Sonuç olarak, topoloji optimizasyon yöntemi kullanılarak parça tasarımında gerçek çalışma şartlarında karşılaşılan yükler ve zorlanmalar karşısında sınır şartları belirlenerek en ekonomik ve optimum ürünler geliştirilebilmektedir.
\end{abstract}

Anahtar Kelimeler: Topoloji optimizasyonu, yapısal optimizasyon, ağırlık azaltma, otomotiv endüstrisi.

\section{A Research on Weight Reduction Application with Topology Optimization in the Automotive Industry}

\begin{abstract}
Recently, the companies in the automotive sector have tended towards the new studies decreasing fuel consumption and increasing energy efficiency in vehicles. One of the most effective solutions for reducing fuel consumption of vehicles in parallel with emission rates is to reduce vehicle weight. Optimization methods are used to ensure the best material distribution in the designed vehicle parts. It is aimed to optimize in size, shape and topology of vehicle parts designed with structural optimization. Structural optimization goals; can be listed as reducing weight, increasing stiffness, reducing stress, ensuring manufacturability, and reducing production costs. Topology optimization is a structural optimization method developed in applied mathematics. Topology optimization finds application in many disciplines such as manufacturing, automotive, construction and aerospace. This article provides an overview of topology optimization and weight reduction efforts in the automotive industry. As an alternative to standard optimization approaches, the topology optimization method is an approach that aims to reduce vehicle weight and find the optimal material distribution in terms of given performance targets. Consequently, the most economic and optimum products can be developed and designed by determining boundary conditions due to the stresses and forces in real time working conditions with using computer software and analysis.
\end{abstract}

Keywords: Topology optimization, structural optimization, weight reduction, automotive industry.

\footnotetext{
* Sorumlu Yazar: fkahraman@ tarsus.edu.tr
} 


\section{Giriş}

Bilgisayar destekli tasarım ve analiz, tüm sanayi dallarında yaygın olarak kullanılmaktadır. Tasarımda ve imalatta bilgisayarların kullanılması artan rekabet koşullarında, ürün özelliklerinin değiştirilmesi ve geliştirilmesi için hızlı tasarım ve üretim sağlamaktadır. Günümüz otomotiv endüstrisinde rekabet şartlarında dayanıklı tasarımlar ile firmalar üstünlük sağlayamamaktadır. Firmalar dayanıklı tasarımlara ek olarak hafif ve maliyeti az olan tasarımlar geliştirmek zorunda kalmıştır. Farklı malzemeler kullanarak daha hafif, daha dayanıklı ve az maliyetli tasarımlar üretilebilmesinin yanı sıra araştırmacılar malzemeyi değiştirmeden belirli kısıtlamalar kullanarak optimum tasarıma ulaşabilecekleri optimizasyon yöntemlerini geliştirmişlerdir.
Tasarımı yapılan taşıt bileşenleri için birçok imalat yöntemi yaygın olarak kullanılmaktadır. Üretime hazır modeller oluşturulurken malzeme dağılımının en iyi şekilde sağlanması için optimizasyon yöntemleri kullanılmaktadır. Optimizasyonun hedefleri Şekil 1'de gösterilmiştir. Mühendisler tasarımlarda stresi artırmadan malzemeyi azaltmanın yöntemlerini araştırmaktadırlar. Optimizasyon teknikleri kullanışlılıkları ve geniş kapsamlı uygulanabilirliklerine karşın iyi bilinmediği ve anlaşılmadığından endüstriyel uygulamaları yeterli değildir (Cavazzuti vd., 2011). Daha verimli ve sürdürülebilir bir imalat için mühendisler tasarımlarında optimizasyon yöntemlerini benimsemelidir. Böylece, ham madde kullanımı, enerji tüketimi ve işletme maliyetleri düşmektedir. Dünyada maden ve enerji kaynakları sınırlıdır ve giderek tükenmektedir. Optimizasyon ile yeterli ham madde kullanımı ve enerji tüketimi kaynakların daha verimli kullanımına katkı sağlayacaktır.

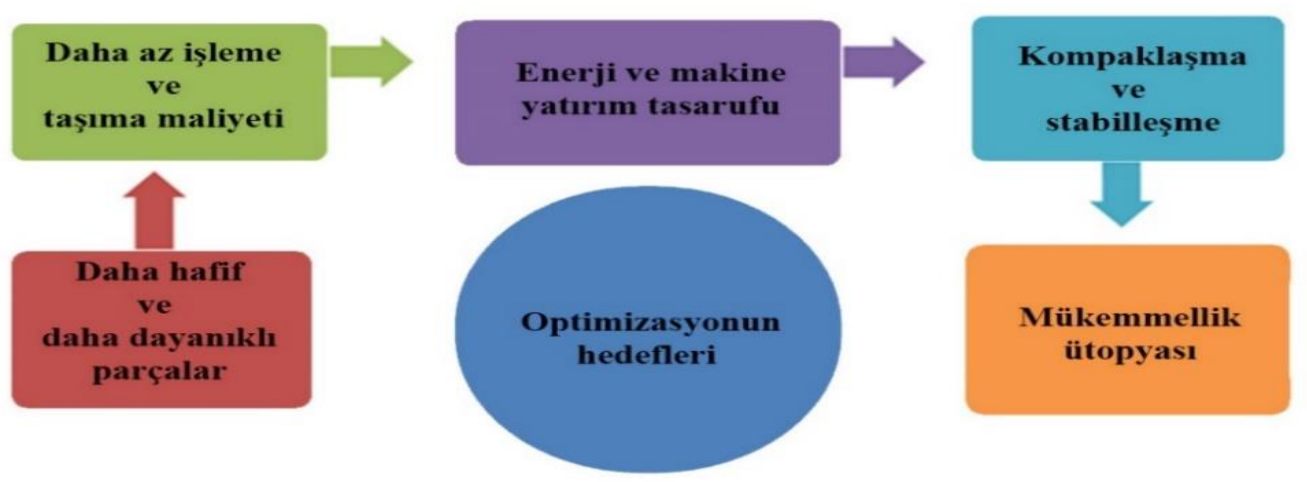

Şekil 1. Optimizasyonun hedefleri (Okudan, 2018)

Optimizasyon yöntemleri iki tür sürece ayrılabilir. İlk süreç, metodolojisi için çoğunlukla matematiksel bir temel kullanan yöntemler diğeri ise, sezgisel metodoloji kullanan yöntemlerdir. Matematiksel optimizasyon algoritmaları matematiksel olarak en doğru çözümü bulma eğilimindeyken, sezgisel yöntemler daha pratik bir yaklaşım kullanır. Yinelemeli bir öğrenme yaklaşımının kullanıldığı sezgisel ve matematiksel yöntemleri temel matematiksel yöntemlerle birleştirmek de mümkündür (Sehmi vd. 2018). Optimizasyon problemlerinin çözümünde doğadaki kanunlardan ve canlıların davranışlarından ilham alan genetik algoritma, yapay arı kolonisi, karınca kolonisi ve parçacık sürü algoritmaları kullanılmaktadır. Optimizasyon bir problemin farklı çözümleri içinden en iyi sonuca ulaştıracak çözümün bulunmasıdır (Çelik vd., 2019). Optimizasyonda, belirli kısıtlamalarda tanımlanan amaç fonksiyonu minimum değere ya da maksimum değere ulaşmalıdır.

Optimizasyon yöntemlerinin kullanımının artması tasarım süreçlerine de yansımıştır. Yapısal optimizasyon ile tasarlanan parçanın topoloji, boyut ve şekil olarak en iyi hale getirilmesi amaçlanır. Topoloji optimizasyonunda parçanın belirlenen bir tasarım alanında optimizasyon uygulanırken boyut ve şekil optimizasyonu parçanın ölçüsü ve şekli değiştirilerek uygulanmaktadır. Yapısal optimzasyonun, topoloji optimizasyonu ile şekil ve boyut optimizasyonu örnekleri Şekil 2'de gösterilmiştir. Yapısal optimizasyonun hedefleri; ağırlık veya hacmi en aza indirmek, sertliği en üst düzeye çıkarmak, stresi azaltmak, üretilebilirliği sağlamak, üretim maliyetlerini düşürmek olarak sıralanabilir.

a)

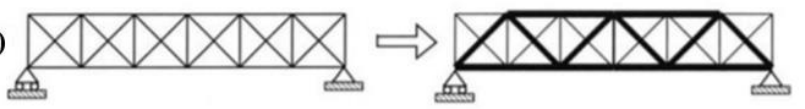

b) $000000 \Rightarrow 0000$

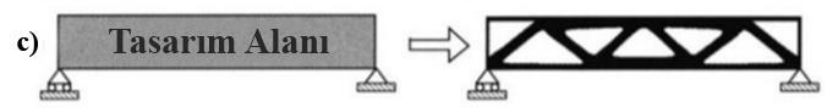

Şekil 2. Yaptsal optimizasyon

a) Boyut optimizasyonu b) Şekil optimizasyonu c) Topoloji optimizasyonu (Bendsoe ve Sigmund, 2003) 


\section{Topoloji Optimizasyonu}

Topoloji optimizasyonu, uygulamalı matematik alanında geliştirlen bir yapısal optimizasyon yöntemidir. Topoloji optimizasyonu makine, malzeme, otomotiv, inşaat ve havacilık mühendisliği gibi birçok disiplinde uygulama alanı bulmaktadır. Topoloji optimizasyonunun temeli bir yapının tasarımına ve sınır kısıtlamalarına göre optimum malzeme dağılımını bulmaktır. Topoloji optimizasyonu hakında ilk makale, 1904 yılında A.G.M. Michell tarafindan yayınlanmıştır (Rozvany, 2009; Tyflopoulos vd., 2018). Modern topoloji optimizasyon yöntemleri için öncü çalışmalar Tablo 1 'de listelenmiş ve topoloji optimizasyon yöntemleri Şekil 3 'te gösterilmiştir.

Tablo 1. Modern topoloji optimizasyon yöntemleri için öncü çalışmaların listesi (Tyflopoulos vd., 2018)

\begin{tabular}{|c|c|}
\hline Yöntemler & Öncü Çalışmalar \\
\hline Homojenizasyon & Bendsoe ve Kikuchi, 1988 \\
\hline \multirow{3}{*}{$\begin{array}{l}\text { SIMP } \\
\text { (Solid Isotropic Microstructure with Penalization) }\end{array}$} & Bendsoe, 1989 \\
\hline & Zhou ve Rozvany, 1991 \\
\hline & Rozvany, 2001 \\
\hline \multirow{3}{*}{$\begin{array}{l}\text { LSA } \\
\text { (Level Set Approach) }\end{array}$} & Osher ve Sethian, 1988 \\
\hline & Michael Wang, Xiaoming Wang ve Guo, 2003 \\
\hline & Jia vd, 2011 \\
\hline \multirow{3}{*}{$\begin{array}{l}\text { ESO } \\
\text { (Evolutionary Structural Optimization) }\end{array}$} & Xie ve Steven, 1993 \\
\hline & Zhou ve Rozvany, 2001 \\
\hline & Xie ve Huang, 2010 \\
\hline \multirow{2}{*}{$\begin{array}{l}\text { BESO } \\
\text { (Bidirectional Evolutionary Structural Optimization) }\end{array}$} & Querin, Young, vd., 2000 \\
\hline & Huang ve Xie, 2007 \\
\hline \multirow{2}{*}{$\begin{array}{l}\text { AESO } \\
\text { (Additive Evolutionary Structural Optimization) }\end{array}$} & Querin, Steven, ve Xie, 1998, 2000 \\
\hline & Querin, Young, Steven, ve Xie, 2000 \\
\hline \multirow{2}{*}{$\begin{array}{l}\text { SKO } \\
\text { (Soft Kill Option) } \\
\end{array}$} & Baumgartner vd., 1992 \\
\hline & Mattheck, 1998 \\
\hline
\end{tabular}

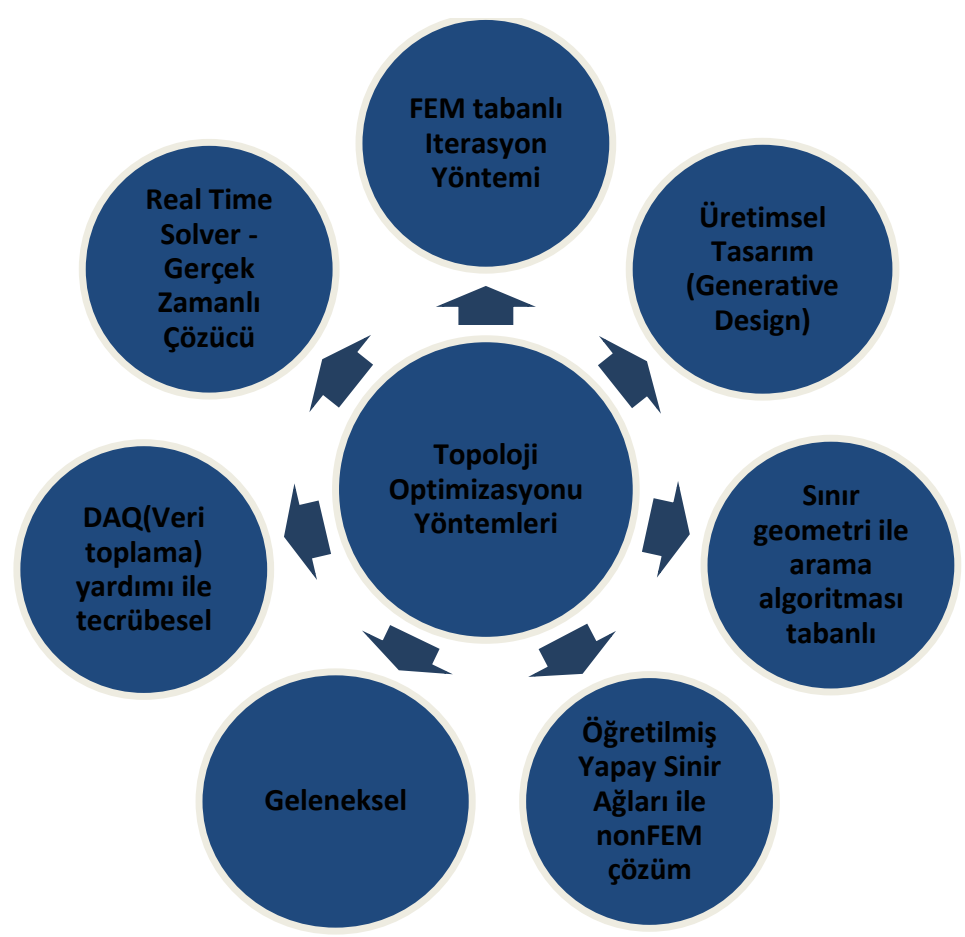

Şekil 3. Topoloji optimizasyonu yöntemleri (Okudan, 2018)

Topoloji optimizasyonu için geliştirilen yazılımlar (TOP, Construct, Catapo, Optcom Optistruct) 1990'lardan itibaren kullanıma başlamıştır. Optimizasyon için kullanılan programlar ve eğitim araçları Tablo 2'de sunulmuştur. 


\section{Avrupa Bilim ve Teknoloji Dergisi}

Tablo 2. Optimizasyon için kullanılan yazılımlar ve eğitim araçları (Reddy vd., 2016)

\begin{tabular}{l|l}
\hline Yazilımlar & Eğitim Araçları \\
\hline Altair Opti Struct & BESO 3D \\
\hline Vanderplaats Genesis & Pareto Works \\
\hline Simulia Tosca & CATOPTO \\
\hline Abaqus ATOM (TOSCA) & Topo struct \\
\hline MSC Nastran & Pro TOp \\
\hline Solid Thinking Inspire & Smart - DO \\
\hline Within Enhance (Autodesk) & META4 ABQ \\
\hline PERMAS - TOPO & To - Py \\
\hline FEM tools Optimization & TRINITAS \\
\hline OPTISHAPE TS & Top - Opt \\
\hline
\end{tabular}

Geometrik modelleme, otomatik ağ oluşturma ve topoloji optimizasyon yöntemlerinin gelişimi, topoloji optimizasyonunun ürün tasarım sürecine entegrasyonuna izin vermekte ve daha geniş kullanımı için koşulları oluşturmaktadır (Şekil 4) (Cuillière vd., 2014). Topoloji optimizasyonu sürecinde tasarım aşamaları Şekil 5 'te gösterilmiştir.

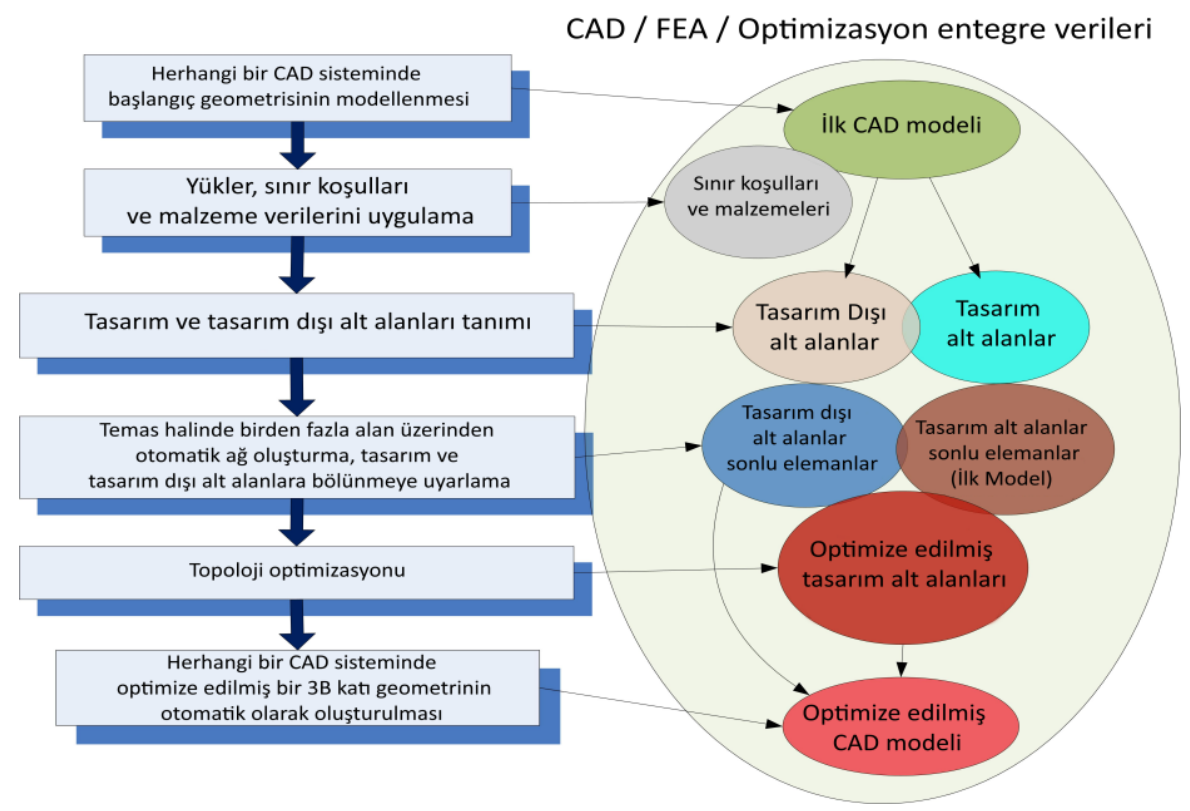

Şekil 4. Topoloji optimizasyonunun tasarım ile entegrasyon şeması (Cuillière vd., 2014)

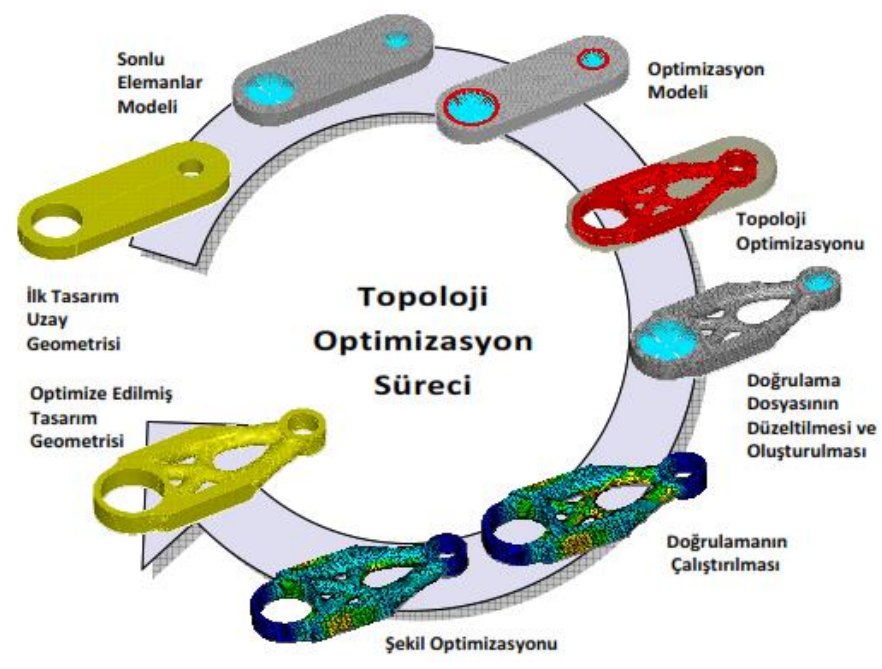

Şekil 5. Topoloji optimizasyonu sürecinde tasarım aşamaları (FEDesign, 2020) 


\section{Otomotiv Endüstrisinde Topoloji Optimizasyonu ile Ağırlık Azaltma}

Günümüzün araç tasarım gereksinimlerini karşılamak, maliyet ve yakıt verimliliğini artırmak için, hafif ve uygun maliyetli araç bileşenlerinin tasarımına olan ilgi giderek artmaktadır. Diğer yandan, ekonomik nedenler ve küresel ısınma nedeniyle otomotiv bileşenlerinin ağırlık olarak azaltılması gerekmektedir. Ağırlık; malzemeler, tasarım tekniği, imalat işlemleri ve optimizasyon gibi çeşitli teknolojik iyileştirmelerle azaltılabilir (Kaya vd., 2010; Zengin ve Köse, 2017). Otomobillerde ağırlığın azaltılması, otomotiv endüstrisinde sık araştırılan bir konudur. Otomotiv endüstrisinde topoloji optimizasyonu yöntemi ile ağırlık azaltma çalışmaları yapılmaktadır (Cavazzuti vd., 2011; Köse ve Mühürcü, 2018).
Güvenlik, yakıt tüketimi gibi verilen performans hedefleri açısından araç ağırlığını azaltılması son derece yararlıdır. 1300 kg ağırlığında bir otomobilde $100 \mathrm{~kg}$ ağırlık azaltıldığında, 100 km'de 0.35 lt yakit tasarrufu ve $8.4 \mathrm{~g} \quad \mathrm{CO} 2 / \mathrm{km} \quad$ egzoz emisyonunda azalma sağlanmaktadır. Başka bir ifadeyle, 100 km'de egzoz emisyonunu $1 \mathrm{~g} \mathrm{CO} 2 / \mathrm{km}$ azaltmak için otomobilin ağırlığı yaklaşık olarak $12 \mathrm{~kg}$ azaltılmalıdır (Meyer-Pruessner, 2007).

Fren pedalının ağırlığını azaltmak için topoloji optimizasyon yöntemi uygulanmaktadır (Albak, 2019; Sudin vd., 2014; Ingale, 2019; Langnau, 2019; Beker, 2020; Top vd., 2019). Fren pedalı için topoloji optimizasyonu örnekleri Şekil 6'da gösterilmiştir.

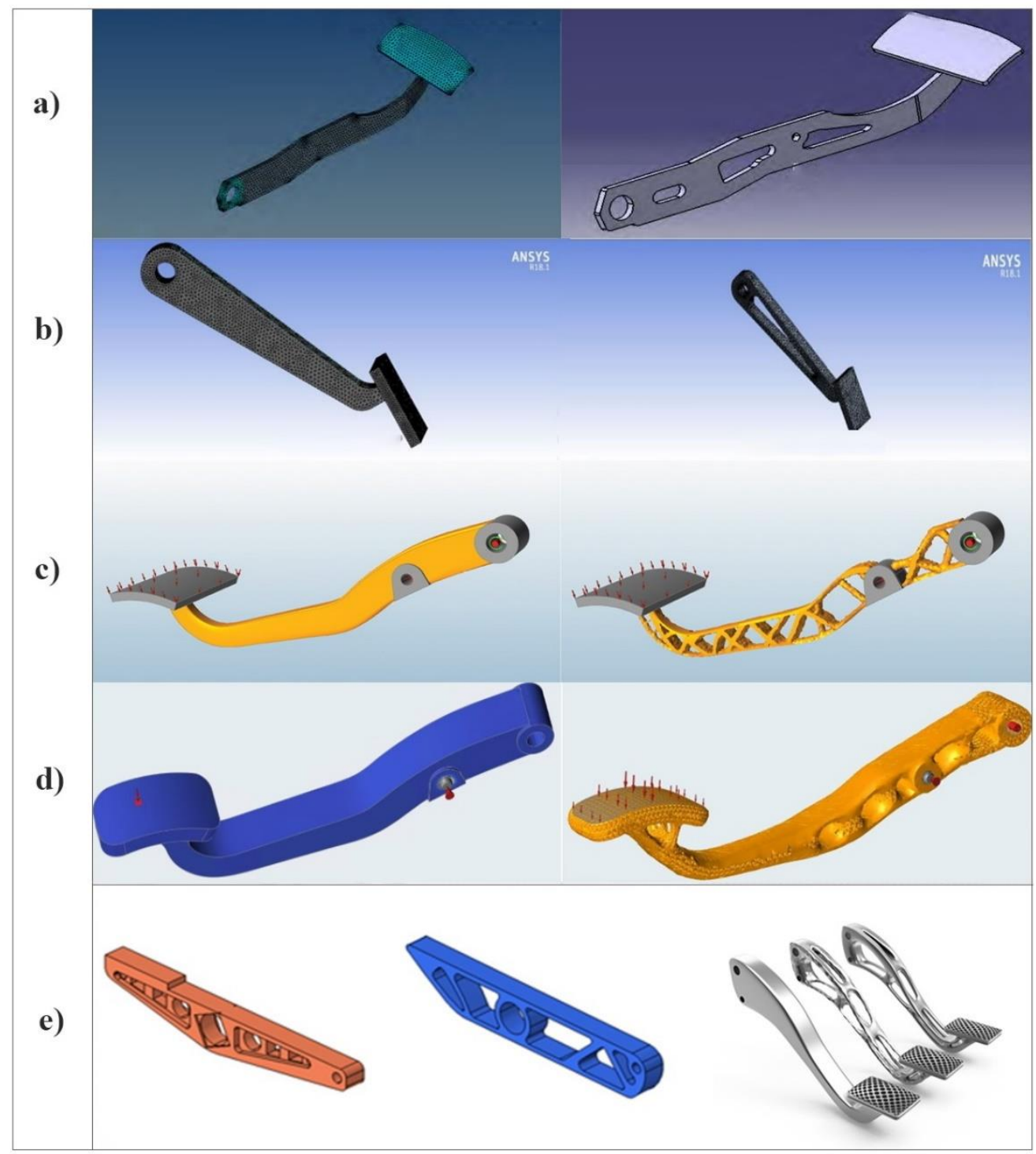

Şekil 6. Fren pedall için topoloji optimizasyonu örnekleri

(a) Sudin vd., 2014 b) Ingale, 2019 c) Langnau, 2019 d) Beker, 2020 e) Albak, 2019)

Güleryüz ve Yılmaz (2019) ağır hizmet taşıtlarında kullanılan kampanalı frenin parçası olan bir tork plakasına topoloji optimizasyonunu uygulamış döküm ve işlenmiş parça ağırlıkları sırasıyla \%11.9 ve \%12.2 azalmıştır.
Jagtap ve Dhoke (2017) üretim kısıtlarını dikkate alarak egzoz montaj braketi için topoloji optimizasyonunu uygulamış ve ağırlığı \%50 oranında azaltmıştır. Topaç vd. (2020) kamyonun arka aksında kullanılan bir bağlantı braketi için 
topoloji optimizasyonunu uygulamış ve parçanın ağırlığı \%63 azaltmıştır. Motor braketinin ağırlığını azaltmak için topoloji optimizasyonu çalışmalarında Öztürk (2016) \%22.6, Wu vd. (2016) \%40 ve Yıldız vd. (2004) \%35 azalma sağlamışlardır.
Deshmukh (2018) taşıt jantlarının ağırlığını azaltmak için topoloji optimizasyonunu uygulamıştır. Taşıt jantı için yapılan topoloji optimizasyonu örneği Şekil 7'de gösterilmiştir.

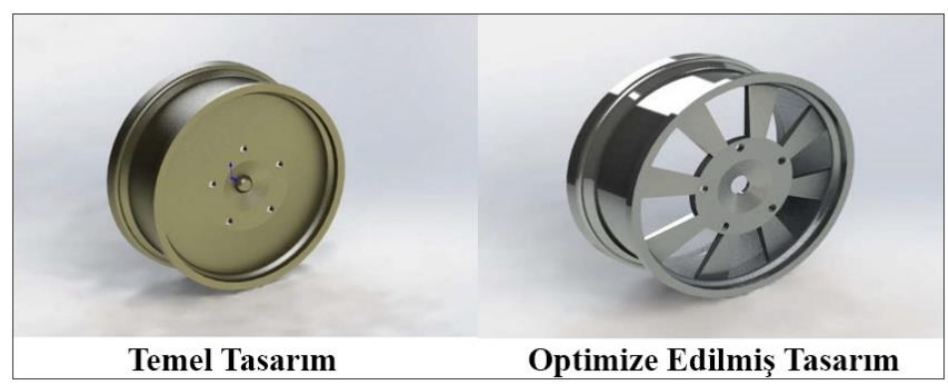

Şekil 7. Jant için topoloji optimizasyonu örneği (Deshmukh, 2018)

Chaudhari ve Khairnar (2020) topoloji optimizasyonu çalışmasında tekerlek göbek bağlantısı için \%24.09 ve mafsal için \%16.30 ağırlık azalması sağlamıştır. Kaya vd. (2010) topoloji ve şekil optimizasyonu ile kavrama çatalını yeniden tasarlamış ve ağırlığı \%24 azaltmıştır. Yenilmez vd. (2019) ve Yaşar vd. (2019) denge çubuğunda topoloji optimizasyonu uygulamışlar her iki çalışmada ağırlığı \%50 azaltmışlardır. Kong vd. (2016) topoloji ve topografya optimizasyonlarını birlikte kullanmış ve helezon yaylı süspansiyon sisteminde kullanılan amortisör alt tablası için yeni tasarımın ağırlığını \%36.5 azaltmıştır. Süspansiyon sisteminde kullanılan kontrol kolunun ağırlığını azaltmak için topoloji optimizasyonu çalışmaları yapılmıştır. Bu yöntemle, Yende vd. (2019) \%16.66, Yıldız vd. (2019) \%18, Detwiler vd. (2015) \%11, Swapnil vd. (2017) $\% 17.5$, Dhore ve Thorat (2019) \%15.40, Y1ld1z (2017) \%28, Topaç vd. (2017) \%19.25 ağırlık azaltımı sağlamışlardır. Topaç vd. (2019) çoklu cisim sistemleri, sonlu elemanlar yöntemi ve topoloji optimizasyonu gibi farklı yaklaşımlar kullanarak süspansiyon sisteminin kavramsal tasarımını yapmıştır. Topoloji optimizasyonu uygulayarak ağırlığı \%37 oranında azaltmıştır. Süspansiyon sisteminde kullanılan kontrol kolu için topoloji optimizasyonu örnekleri Şekil 8'de gösterilmiştir.

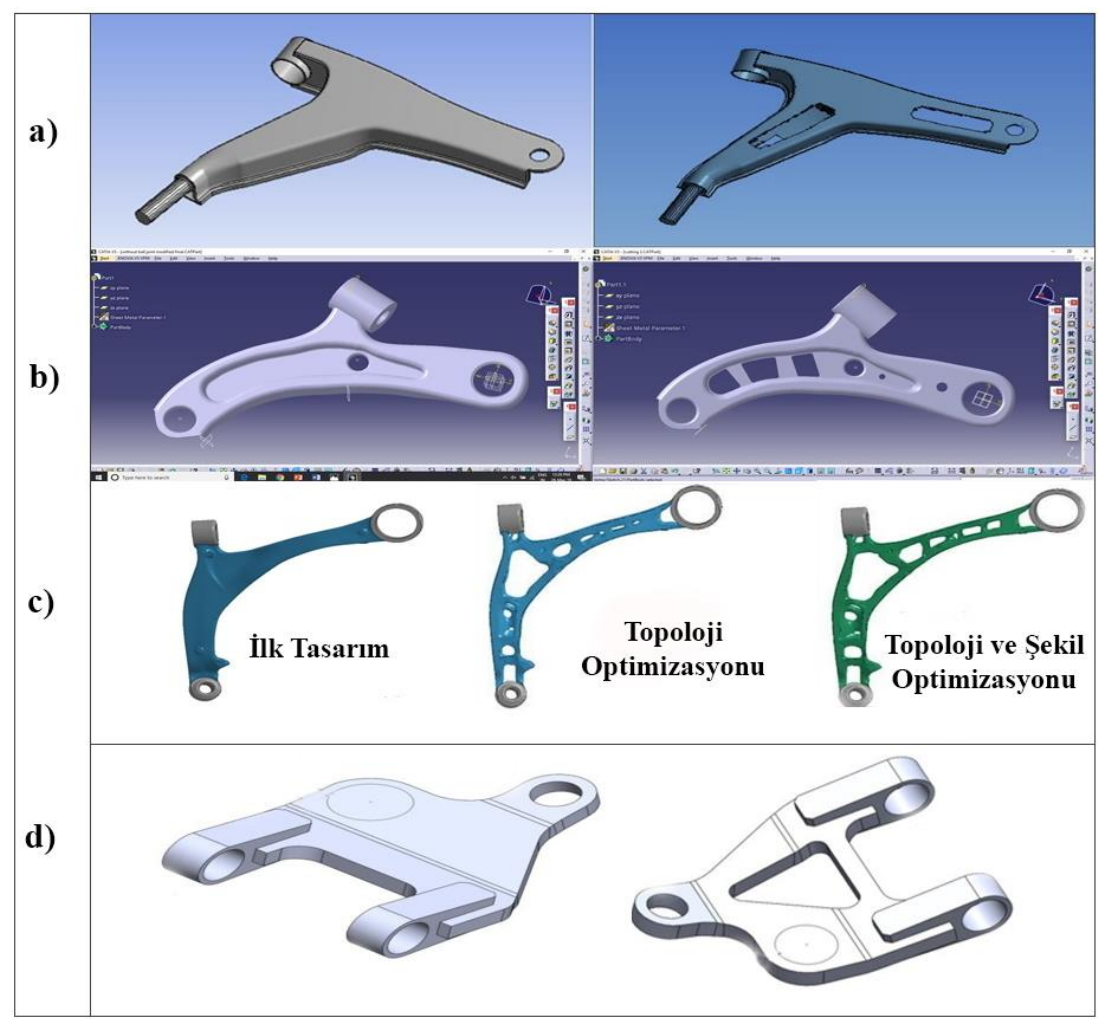

Şekil 8. Süspansiyon kontrol kolu için topoloji optimizasyonu örnekleri

(a) Dhore ve Thorat, 2019 b) Yende vd., 2019 c) Detwiler vd., 2015 d) Topaç vd., 2017) 
Mantovani vd. (2017) bir spor otomobil gösterge paneline topoloji optimizasyonunu uygulamıştır. Karaoğlan vd. (2016) hidrostatik tahrikli bir yol yıkama aracında optimizasyon çalışması ile uygun profil et kalınlığının belirlenmesi ve şasi üzerindeki gerilmenin az olduğu yerlerden ağırlığın azaltması sonucu şaside \%18.81 hafifleme sağlamıştır. Koçar vd. (2018) 26 ton kapasiteli açık kasa kuru yük dorse şasi ana taşıyıcısını incelemiş ve toplam ağırlığın 475.98 kg'dan 280 kg kadar azaltılabileceğini belirlemiştir. Höke ve Bozca (2019) sac levhadan üretilen motor traverslerinin tasarımında ağırlığ azaltmak için topoloji-topografya optimizasyonu bileşimi ve deneme-yanılma yöntemlerini kullanmışlardır. Yıldız (2012) otomotiv endüstrisinde tasarım optimizasyon problemini çözmek için Taguchi tasarım yaklaşımı ve parçacık sürü optimizasyon yöntemine dayanan yeni bir yaklaşım sunmuştur.

\section{Sonuç}

Otomotiv, makine, imalat ve havacılık gibi birçok üretim alanında; kalite iyileştirme, malzeme tasarrufu, zaman ve maliyet verimliliği için ürünlerin optimizasyonu büyük önem taşımaktadır. Yapısal optimizasyon ile tasarlanan taşıt parçalarının; boyut, şekil ve topoloji olarak en iyi hale getirilmesi amaçlanmaktadır Araçların yakıt tüketimini ve buna bağlı olarak da emisyon oranlarını azaltmada en etkili çözümlerden biri taşıt ağırlığında azaltılma yapılmasıdır. Günümüzde otomotiv endüstrisinde topoloji optimizasyon yöntemi ile ağırlık azaltma çalışmaları geniş çapta yapılmaktadır Topolojik optimizasyonun avantajları;

- Hafif ve üretime hazır ürünlerin oluşturulması,

- Ürünün analizlerinin bilgisayar ortamında gerçekleştirilmesini sağlayarak, test süreçlerini kısaltması

- Gerçek yük ve kısıtlamalar ile çalışması olarak siralanabilir.

Sonuç olarak, topoloji optimizasyon yöntemi kullanılarak parça tasarımında gerçek çalışma şartlarında karşılaşılan yükler ve zorlanmalar karşısında sınır şartları belirlenerek en ekonomik ve optimum ürünler geliştirilebilmektedir.

\section{Kaynakça}

Albak, İ. (2019). Optimum design of brake pedal using topology optimization method intended for weight reduction on the formula SAE car. Uluslararası Mühendislik Araştırma Ve Gelistirme Dergisi, 328-334. doi:10.29137/umagd.467057

Baumgartner, A., Harzheim, L., \& Mattheck, C. (1992). SKO (soft kill option): the biological way to find an optimum structure topology. International Journal of Fatigue, 14(6), 387-393. doi:10.1016/0142-1123(92)90226-3

Beker, S., (2020). Topoloji Optimizasyonu Yaklaşımıyla Ürün Geliştirme, 01.11.2020 tarihinde https://www.poligonmu hendislik. com/blog/urun-gelistirme/topoloji-optimizasyonu -yaklasimi-ile-urun-gelistirme adresinden edinilmiştir.

Bendsøe, M. P. (1989). Optimal shape design as a material distribution problem. Structural Optimization, 1(4), 193202. doi:10.1007/bf01650949

Bendsøe, M. P., ve Kikuchi, N. (1988). Generating optimal topologies in structural design using a homogenization method. Computer Methods in Applied Mechanics and Engineering, 71(2), 197-224. doi:10.1016/0045-7825(88) 90086-2
Bendsoe, M. P., \& Sigmund, O. (2003). Topology optimization: theory, methods, and applications. Springer Science \& Business Media.

Cavazzuti, M., Baldini, A., Bertocchi, E., Costi, D., Torricelli, E. ve Moruzzi, P. (2011). High performance automotive chassis design: a topology optimization based approach. Structural and Multidisciplinary Optimization, 44(1), 45-56.

Chaudhari, P. ve Khairnar, R. (2020). Weight optimization of hub and knuckle using topology optimization. International Journal of Mechanical Engineering, 7(6), 20-23. doi:10.14445/23488360/ijme-v7i6p103

Cuillière, J., Francois V., ve Drouet, J., (2014). Towards the Integration of Topology Optimization into the CAD Process, Computer-Aided Design and Applications, 11:2, 120-140, DOI:10.1080/16864360.2014.846067

Çelik, Y. Yıldız, İ. Karadeniz, A.T. (2019). A Brief Review of Metaheuristic Algorithms Improved in the Last Three Years. Avrupa Bilim ve Teknoloji Dergisi, (Özel Say1), 463-477.

Deshmukh, P., (2018). Topology Optimization of wheel rim of Heavy Vehicle, International Journal of Current Advanced Research, 07(6), pp. 13289-13291. DOI:http://dx.doi.org /10.24327/ijcar.2018.13291.2362

Detwiler, D., Nutwell, E., Lokesha, D., (2015). Multistage optimization of automotive control arm through topology and shape optimization. 6th BETA CAE International Conference, 10-12 June 2015, Greece.

Dhore, R., Thorat, M.L, (2019). Experımental analysis and topology optimization of lower suspension arm of car. International Research Journal of Engineering and Technology (IRJET), 06(03), 7605-7609.

FEDesign, (2020). Design Process with ANSYS Workbench and TOSCA Structure https://support.ansys.com/staticassets/ ANSYS/ staticassets/ partner/ FEDesign/ fedesign- toscabrochure.pdf adresinden edinilmiştir.

Güleryüz, İ. ve Yılmaz, B. (2019). Ağır hizmet araçlarında kullanılan Z-kam kampanalı fren tork plakası ağırlık optimizasyonu. Academic Perspective Procedia, 2(3), 466475.

Höke, Ö. ve Bozca, M. (2019). Topology Optimisation of Engine Cross Members for Lightweight Structure in Light Commercial Vehicles. International Journal of Precision Engineering and Manufacturing, 21(3), 465-482. doi:10.1007/s12541-019-00228-4

Huang, X., ve Xie, Y. M. (2007). Convergent and meshindependent solutions for the bi-directional evolutionary structural optimization method. Finite Elements in Analysis and Design, 43(14), 1039-1049. doi:10.1016/j.finel.2007 .06 .006

Ingale, P.M. (2019). Topology Optimization of an All-Terrain Vehicle Brake Pedal. International Journal of Engineering Research \& Technology (IJERT), 8(6), 763-767.

Jagtap, M. ve Dhoke, A. (2017). Topology optimization of exhaust mounting bracket. In Tech Mahindra, Altair Technology Conference.

Jia, H., Beom, H. G., Wang, Y., Lin, S., ve Liu, B. (2011). Evolutionary level set method for structural topology optimization. Computers \& Structures, 89(5-6), 445-454. doi:10.1016/j.compstruc.2010.11.003

Karaoğlan, M. U., Turnalı, Ö. Y. ve Kuralay, S. N. (2016). Hafif hizmet aracı şasi analizi ve optimizasyonu. Deu Mühendislik Fakültesi Fen ve Mühendislik, 18(54), 502502. doi:10.21205/deufmd.2016185417 
Kaya, N., Karen, İ. ve Öztürk, F. (2010). Re-design of a failed clutch fork using topology and shape optimisation by the response surface method. Materials and Design, 31(6), 3008-3014. doi:10.1016/j.matdes.2010.01.002

Koçar, O., Şen, Ş., Yaşar, M. (2018). Dorse tasarımında stres dağılım analizi ve topoloji optimizasyonu. Karaelmas Science and Engineering Journal, 8(1), 309-316.

Kong, Y. S., Abdullah, S., Omar, M. Z. ve Haris, S. M. (2016). Topological and topographical optimization of automotive spring lower seat. Latin American Journal of Solids and Structures, 13(7), 1388-1405. doi:10.1590/1679-78252082

Köse, E., ve Mühürcü, A. (2018). Çeşitli kriterlere göre elektrikli otomobillerin karşılaştırılması. Presented at the 3rd International Mediterranean Science and Engineering Congress (IMSEC 2018), ADANA

Langnau, L., (2019). What is topology optimization? 1.11.2020 tarihinde https://www.makepartsfast.com/what-is-topologyoptimization adresinden edinilmiştir.

Mantovani, S., Presti, I. L., Cavazzoni, L. ve Baldini, A. (2017). Influence of manufacturing constraints on the topology optimization of an automotive dashboard. Procedia Manufacturing, 11, 1700-1708.

Mattheck, C. (1998). Design in der Natur und nach der Natur. Kreativität Als Chance Für Den Standort Deutschland, 6370. doi:10.1007/978-3-642-60292-4_9

Meyer-Pruessner, R. (2007). Significant weight reduction by using topology optimization in volkswagen design development with emphasis on engine design [PDF Belgesi] 21.07.2020 tarihinde https://www.altairhyperworks.co.uk/ html/en-GB/session1/Meyer-Pr\%FCessner_Volkswagen.pdf adresinden edinilmişstir.

Okudan, A. (2018, 6 Eylül). Topoloji optimizasyonu-101. 09.08.2020 tarihinde https://tr.linkedin.com/pulse/topolojioptimizasyonu-101-ahmet-okudan adresinden edinilmiştir.

Osher, S., ve Sethian, J. A. (1988). Fronts propagating with curvature-dependent speed: Algorithms based on HamiltonJacobi formulations. Journal of Computational Physics, 79(1), 12-49. doi:10.1016/0021-9991(88)90002-2

Öztürk, U. E. (2016). Döküm braketlerin bilgisayar destekli tasarım eniyilemesi için yeni bir yaklaşım. Deu Mühendislik Fakültesi Fen ve Mühendislik, 18(54), 521521. doi:10.21205/deufmd.2016185419

Querin, O. M., Steven, G. P. ve Xie, Y. M. (2000). Evolutionary structural optimisation using an additive algorithm. Finite Elements in Analysis and Design, 34(3-4), 291-308. doi:10.1016/s0168-874x(99)00044-X

Querin, O. M., Steven, G. P., ve Xie, Y. M. (1998). Evolutionary structural optimisation (ESO) using a bidirectional algorithm. Engineering Computations, 15(8), 1031-1048. doi:10.1108/ 02644409810244129

Querin, O. M., Young, V., Steven, G. P. ve Xie, Y. M. (2000). Computational efficiency and validation of bi-directional evolutionary structural optimisation. Computer Methods in Applied Mechanics and Engineering, 189(2), 559-573. doi:10.1016/s0045-7825(99)00309-6

Reddy K, S. N., Ferguson, I., Frecker, M., Simpson, T. W., ve Dickman, C. J. (2016, August). Topology optimization software for additive manufacturing: A review of current capabilities and a real-world example. In International Design Engineering Technical Conferences and Computers and Information in Engineering Conference (Vol. 50107, p. V02AT03A029). American Society of Mechanical Engineers.
Rozvany, G. I. N. (2001). Aims, scope, methods, history and unified terminology of computer-aided topology optimization in structural mechanics. Structural and Multidisciplinary Optimization, 21(2), 90-108. doi:10.1007/ s001580050174

Rozvany, G. I. N. (2008). A critical review of established methods of structural topology optimization. Structural and Multidisciplinary Optimization, 37(3), 217-237. doi:10.1007/s00158-007-0217-0

Sehmi, M., Christensen, J., Bastien, C. ve Kanarachos, S. (2018). Review of topology optimisation refinement processes for sheet metal manufacturing in the automotive industry. Structural and Multidisciplinary Optimization, 58(1), 305-330.

Sudin, M. N., Tahir, M. M., Ramli, F. R. ve Shamsuddin, S. A. (2014). Topology optimization in automotive brake pedal redesign. International Journal of Engineering and Technology (IJET), 6(1), 398-402.

Swapnil, S. K., Amol, N. P., ve Amol, B. G. (2017). Design optimisation of a lower control arm of suspension system in a LCV by using topological approach. International Journal of Innovative Research in Science, Engineering and Technology, 6(6), 11657-11665.

Top, N., Gökçe, H. ve Şahin, İ. (2019). Eklemeli imalat için topoloji optimizasyonu: el freni mekanizması uygulaması. Selçuk-Teknik Dergisi, 18(1), 1-13

Topaç, M. M., Bahar, E., Kaplan, A. ve Sarıkaya, E. Z. (2017). Topoloji optimizasyonu yardımıyla, askeri taşıt bağımsız ön süspansiyonu için alt salıncak tasarımı. IDEFIS 2017: 2nd International Defence Industry Symposium 333-342

Topaç, M. M., Karaca, M., Aksoy, B., Deryal, U. ve Bilal, L. (2020). Lightweight design of a rear axle connection bracket for a heavy commercial vehicle by using topology optimisation: A case study. Mechanics, 26(1), 64-72.

Topaç, M. M., Özmen, B., Deryal, U. ve Selbes, O. (2019). Özel tip bir yarı römork için bağımsız süspansiyon sistemi tasarımı: Kavramsal tasarım çalışmaları. Journal of Polytechnic. doi:10.2339/politeknik.399078

Tyflopoulos, E., Tollnes, F. D., Steinert, M. ve Olsen, A. (2018). State of the art of generative design and topology optimization and potential research needs. DS 91: Proceedings of NordDesign 2018, Linköping, Sweden, 14th17th August 2018.

Wang, M. Y., Wang, X., ve Guo, D. (2003). A level set method for structural topology optimization. Computer Methods in Applied Mechanics and Engineering, 192(1-2), 227-246. doi:10.1016/s0045-7825(02)00559-5

Wu, P., Ma, Q., Luo, Y., ve Tao, C. (2016). Topology optimization design of automotive engine bracket. Energy and Power Engineering, 8(04), 230.

Xie, Y. M., ve Huang, X. (2010). Recent developments in evolutionary structural optimization (ESO) for continuum structures. IOP Conference Series: Materials Science and Engineering, 10, 012196. doi:10.1088/1757-899x/10/1/ 012196

Xie, Y. M., ve Steven, G. P. (1993). A simple evolutionary procedure for structural optimization. Computers \& Structures, 49(5), 885-896. doi:10.1016/0045-7949(93) 90035-c

Yaşar, A., Yılmaz, E., Şendur, P., (2019) Şantiye Kamyonlarında Denge Çubuğu Tasarımı ve Optimizasyonu. Mühendis ve Makina Güncel, Sayı32, 2-32. 
Yende, S. V., Tadamalle, A. P., ve Burande, D. H. (2019). Topology Optimization of Lower Control Arm for LMV. International Journal of Engineering Research \& Technology (IJERT), 08(07), 829-834.

Yenilmez, E., Yasar, A. ve Şendur, P. (2019). Şantiye kamyonlarında denge çubuğu tasarımı ve optimizasyonu, Mühendis ve Makina Güncel, Ağustos 2019, Say1:32, 28-32.

Yildiz, A. R. (2012). A new hybrid particle swarm optimization approach for structural design optimization in the automotive industry, Proceedings of the Institution of Mechanical Engineers, Part D: Journal of Automobile Engineering, 226 (10), 1340-1351.

Yıldız, A. R. (2017). Taşıt elemanlarının yapısal optimizasyon teknikleri ile optimum tasarımı. Politeknik Dergisi, 20(2), 319-323.

Yıldız, A. R., Kaya, N., Öztürk, F. ve Alankuş, O. (2004). Optimal design of vehicle components using topology design and optimisation. International Journal of Vehicle Design, 34(4), 387. doi:10.1504/ijvd.2004.004064
Yıldız, A. R., Kılıçarpa, U. A., Demirci, E. ve Doğan, M. (2019). Topography and topology optimization of diesel engine components for light-weight design in the automotive industry. Materials Testing, 61(1), 27-34. doi:10.3139/120. 111277

Zengin, E., ve Köse, E. (2017). Standart bir otomobilin elektrikli otomobile dönüşümü üzerine gerçek bir uygulama. Presented at the 2nd International Mediterranean Science and Engineering Congress (IMSEC 2017), ADANA.

Zhou, M., ve Rozvany, G. I. N. (1991). The Coc Algorithm .2. Topological, Geometrical and Generalized Shape Optimization. Computer Methods in Applied Mechanics and Engineering, 89(1-3), 309-336. doi:Doi 10.1016/0045-7825 (91)90046-9

Zhou, M., ve Rozvany, G. I. N. (2001). On the validity of ESO type methods in topology optimization. Structural and Multidisciplinary Optimization, 21(1), 80-83. doi:10.1007/s 001580050170 